TRANSACTIONS OF THE

AMERICAN MATHEMATICAL SOCIETY

Volume 353, Number 5, Pages 1767-1779

S 0002-9947(01)02726-X

Article electronically published on January 5, 2001

\title{
A NEW AFFINE INVARIANT FOR POLYTOPES AND SCHNEIDER'S PROJECTION PROBLEM
}

\author{
ERWIN LUTWAK, DEANE YANG, AND GAOYONG ZHANG
}

\begin{abstract}
New affine invariant functionals for convex polytopes are introduced. Some sharp affine isoperimetric inequalities are established for the new functionals. These new inequalities lead to fairly strong volume estimates for projection bodies. Two of the new affine isoperimetric inequalities are extensions of Ball's reverse isoperimetric inequalities.
\end{abstract}

If $K$ is a convex body (i.e., a compact, convex subset with nonempty interior) in Euclidean $n$-space, $\mathbb{R}^{n}$, then on the unit sphere, $S^{n-1}$, its support function, $h(K, \cdot): S^{n-1} \rightarrow \mathbb{R}$, is defined for $u \in S^{n-1}$ by $h(K, u)=\max \{u \cdot y: y \in K\}$, where $u \cdot y$ denotes the standard inner product of $u$ and $y$. The projection body, $\Pi K$, of $K$ can be defined as the convex body whose support function, for $u \in S^{n-1}$, is given by

$$
h(\Pi K, u)=\operatorname{vol}_{n-1}\left(K \mid u^{\perp}\right),
$$

where $\operatorname{vol}_{n-1}$ denotes $(n-1)$-dimensional volume and $K \mid u^{\perp}$ denotes the image of the orthogonal projection of $K$ onto the codimension 1 subspace orthogonal to $u$.

An important unsolved problem regarding projection bodies is Schneider's projection problem: What is the least upper bound, as $K$ ranges over the class of origin-symmetric convex bodies in $\mathbb{R}^{n}$, of the affine-invariant ratio

$$
\left[V(\Pi K) / V(K)^{n-1}\right]^{1 / n},
$$

where $V$ is used to abbreviate $\operatorname{vol}_{n}$. See $[\underline{\mathrm{S}}$, $[\mathrm{S2}$, $[\mathrm{SW}]$ and [Le]. Schneider $\mathrm{S} 1$ conjectured that this ratio is maximized by parallelotopes. In [S1], Schneider also presented applications of such results in stochastic geometry. However, a counterexample was produced in $\mathrm{Br}$ to show that this is not the case.

We will present a modified version of Schneider's conjecture that has an affirmative answer. In addition, we will obtain an inequality that gives an upper bound for the affine ratio $(*)$. While our upper bound is not sharp for any $n$, nevertheless it is asymptotically optimal.

To be more specific, in this paper, we introduce a new centro-affine functional $U$, defined on the class of polytopes, which is closely related to the volume functional $V$. While in general $U(K)<V(K)$, if $K$ is a random polytope (with many faces), then $U(K)$ is very close to $V(K)$. We shall prove the following variation of Schneider's projection conjecture:

Received by the editors February 26, 2000.

1991 Mathematics Subject Classification. Primary 52A40.

Key words and phrases. Affine isoperimetric inequalities, reverse isoperimetric inequalities, projection bodies, asymptotic inequalities.

Research supported, in part, by NSF Grant DMS-9803261. 
Theorem. If $K$ is an origin-symmetric convex polytope in $\mathbb{R}^{n}$, then

$$
\frac{V(\Pi K)}{U(K)^{\frac{n}{2}} V(K)^{\frac{n}{2}-1}} \leq 2^{n}\left(\frac{n^{n}}{n !}\right)^{\frac{1}{2}}
$$

with equality if and only if $K$ is a parallelotope.

The inequality of the theorem immediately provides an asymptotically optimal bound for the affine ratio $(*)$ :

Corollary 4.7. If $K$ is a convex body in $\mathbb{R}^{n}$ that is symmetric about some point, then

$$
V(\Pi K) / V(K)^{n-1} \leq 2^{n}\left(\frac{n^{n}}{n !}\right)^{\frac{1}{2}} .
$$

While the inequality of Corollary 4.7 is not sharp for any value of $n$, it is asymptotically optimal in the sense that a weakened form of Corollary 4.7 is

Corollary $4.7^{-}$. If $K$ is a convex body in $\mathbb{R}^{n}$ that is symmetric about some point, then

$$
\left[V(\Pi K) / V(K)^{n-1}\right]^{1 / n} \leq 2 \sqrt{e} .
$$

If $K$ is taken to be the cube, then the affine ratio $(*)$ is a constant (to be specific 1 ) independent of the dimension $n$. Thus, up to a constant multiple, the inequality of Corollary $4.7^{-}$is best possible. The fact that there exists a constant, independent of the dimension $n$, that dominates the affine ratio $(*)$ was shown by Giannopoulos and Papadimitrakis GiPa.

We will also establish a sharp affine isoperimetric inequality (Theorem 4.11) for our new functional that will immediately give:

Corollary 4.12. If $K$ is a convex body in $\mathbb{R}^{n}$, then

$$
V(\Pi K) / V(K)^{n-1} \leq n^{n}(n+1)^{\frac{n+1}{2}}(n !)^{-\frac{3}{2}} .
$$

Again, while this inequality is not sharp for any value of $n$, it is asymptotically optimal.

Yet a third sharp affine isoperimetric inequality (Theorem 4.8) for our functional will yield an asymptotically optimal bound for an open problem regarding polar projection bodies (Corollary 4.9).

In the next-to-last section, we introduce a family of affine functionals, $U_{1}, \ldots, U_{n}$, for which $U_{1}=V$ and $U_{n}=U$. Two sharp affine isoperimetric inequalities (Theorems 5.2 and 5.3) will be presented for these functionals. These inequalities generalize Ball's reverse isoperimetric inequality.

\section{BACKGROUND AND NOTATION}

In this section we present the terminology and notation we shall use throughout. For quick reference we collect some known results that will be the ingredients of the proofs given in subsequent sections. For general reference the reader may wish to consult the books of Gardner G], Leichtweiß [Le, Schneider [S2], and Thompson T.

If $K$ is a convex body that contains the origin in its interior, then write $K^{*}$ for the polar of $K$; i.e.,

$$
K^{*}=\left\{x \in \mathbb{R}^{n}: x \cdot y \leq 1 \text { for all } y \in K\right\} .
$$


Let $P$ be a convex polytope in $\mathbb{R}^{n}$ that contains the origin in its interior. Let $u_{1}, \ldots, u_{N}$ denote the outer unit normals of $P$. Let $h_{1}, \ldots, h_{N}$ denote the corresponding distances from the origin to the faces and $a_{1}, \ldots, a_{N}$ the areas (i.e. $(n-1)$-dimensional volumes) of the corresponding faces. In [LYZ the ellipsoid $\Gamma_{-2} P$ was defined as the ellipsoid whose polar, $\Gamma_{-2}^{*} P$, has its support function given by

$$
h\left(\Gamma_{-2}^{*} P, u\right)^{2}=\frac{1}{V(P)} \sum_{i=1}^{N}\left|u \cdot u_{i}\right|^{2} \frac{a_{i}}{h_{i}},
$$

for $u \in S^{n-1}$. Note that we use $\Gamma_{-2}^{*} P$ rather than $\left(\Gamma_{-2} P\right)^{*}$ to denote the polar of $\Gamma_{-2} P$. The new ellipsoid is in a sense a dual of the Legendre ellipsoid of classical mechanics. (See e.g., Leichtweiß [Le, Lindenstrauss and Milman [LiM], Milman and Pajor [MPa1], [MPa2, and Petty [P1] for reference regarding the Legendre ellipsoid.)

We shall make use of the fact that the operator $\Gamma_{-2}$ is a centro-affine operator in the sense that

$$
\Gamma_{-2} \phi P=\phi \Gamma_{-2} P, \quad \text { for all } \phi \in \mathrm{GL}(n),
$$

where $\phi P=\{\phi x: x \in P\}$. This fact was established in [LYZ]. We shall also require a similar fact, first established by Petty $\mathrm{P} 2$ (see e.g. BoLi] and $\mathrm{L}$, for alternate proofs), regarding the operator $\Pi$ :

$$
\Pi \phi P=\phi^{-t} \Pi P, \quad \text { for all } \phi \in \mathrm{SL}(n),
$$

where $\phi^{-t}$ denotes the inverse of the transpose of $\phi$. We shall write $\Pi^{*} P$ for the polar of $\Pi P$, rather than $(\Pi P)^{*}$.

Recall that McMullen's intrinsic volumes, $V_{0}(P), \ldots, V_{n}(P)$, of the polytope $P$ can be defined [Mc] as coefficients in the Steiner polynomial:

$$
V(P+\lambda B)=\sum_{i=0}^{n} \lambda^{i} \omega_{i} V_{n-i}(P),
$$

where $\omega_{i}$ is the $i$-dimensional volume of the unit ball in $\mathbb{R}^{i}$ and $\omega_{0}=1$. Thus $V_{n}(P)=V(P)$.

Suppose $u_{1}, \ldots, u_{N} \in S^{n-1}$ and $\lambda_{1}, \ldots, \lambda_{N}>0$. If $K$ is a convex body whose support function, for $u \in S^{n-1}$, is given by

$$
h(K, u)=\sum_{i=1}^{N} \lambda_{i}\left|u \cdot u_{i}\right|
$$

then $K$ is called a zonotope. Obviously the projection bodies of polytopes are zonotopes. Although we shall make no use of this fact, it can be shown that all zonotopes are projection bodies of origin-symmetric polytopes. We will need the McMullen-Matheron-Weil formula (see [Sh], [Ma], and [W]) for the intrinsic volume, $V_{k}(K)$, for $1 \leq k \leq n$, of the zonotope $K$ :

$$
V_{k}(K)=\frac{2^{k}}{k !} \sum_{1 \leq i_{1}, \ldots, i_{k} \leq N} \lambda_{i_{1}} \cdots \lambda_{i_{k}}\left[u_{i_{1}}, \ldots, u_{i_{k}}\right]
$$

where $\left[u_{i_{1}}, \ldots, u_{i_{k}}\right]$ denotes the $k$-dimensional volume of the $k$-dimensional parallelotope $\left\{c_{1} u_{i_{1}}+\cdots+c_{k} u_{i_{k}}: 0 \leq c_{i} \leq 1\right\}$. (See [SW] and GoW] for surveys about zonoids and zonotopes.) 
The John ellipsoid of a convex body is the largest (in volume) ellipsoid that is contained in the body. The John point of a convex body is the center of the John ellipsoid of the body. A convex body in $\mathbb{R}^{n}$ is said to be in John position if its John ellipsoid is the standard unit ball in $\mathbb{R}^{n}$. Obviously, every convex body in $\mathbb{R}^{n}$ may be $\mathrm{GL}(n)$-transformed into John position.

\section{A BASIC IDENTITY}

The following basic fact is critical for our main results.

Lemma 2.1. Suppose $u_{1}, \ldots, u_{N} \in S^{n-1}$ and $\lambda_{1}, \ldots, \lambda_{N}>0$. If

$$
\sum_{i=1}^{N} \lambda_{i}\left|u \cdot u_{i}\right|^{2}=1, \quad \text { for all } u \in S^{n-1},
$$

then for each $k$ such that $1 \leq k \leq n$,

$$
\sum_{1 \leq i_{1}, \ldots, i_{k} \leq N} \lambda_{i_{1}} \cdots \lambda_{i_{k}}\left[u_{i_{1}}, \ldots, u_{i_{k}}\right]^{2}=\frac{n !}{(n-k) !}
$$

To prove Lemma 2.1 we shall make use of some basic facts regarding mixed discriminants. Recall that for positive semi-definite $n \times n$ matrices $Q_{1}, \ldots, Q_{N}$ and real $\lambda_{1}, \ldots, \lambda_{N} \geq 0$, the determinant of the linear combination $\lambda_{1} Q_{1}+\cdots+\lambda_{N} Q_{N}$ is a homogeneous polynomial of degree $n$ in the $\lambda_{i}$,

$$
\operatorname{det}\left(\lambda_{1} Q_{1}+\cdots+\lambda_{N} Q_{N}\right)=\sum_{1 \leq i_{1}, \ldots, i_{n} \leq N} \lambda_{i_{1}} \cdots \lambda_{i_{n}} D\left(Q_{i_{1}}, \ldots, Q_{i_{n}}\right),
$$

where the coefficient $D\left(Q_{i_{1}}, \ldots, Q_{i_{n}}\right)$ depends only on $Q_{i_{1}}, \ldots, Q_{i_{n}}$ (and not on any of the other $Q_{j}$ ) and thus may be chosen to be symmetric in its arguments. The coefficient $D\left(Q_{i_{1}}, \ldots, Q_{i_{n}}\right)$ is called the mixed discriminant of $Q_{i_{1}}, \ldots, Q_{i_{n}}$.

The mixed discriminant $D(Q, \ldots, Q, I, \ldots, I)$, with $k$ copies of $Q$ and $n-k$ copies of the identity matrix, $I$, will be abbreviated by $D_{k}(Q)$. Note that the elementary mixed discriminants $D_{0}(Q), \ldots, D_{n}(Q)$ are thus defined as the coefficients of the polynomial

$$
\operatorname{det}(Q+\lambda I)=\sum_{i=0}^{n}\left(\begin{array}{l}
n \\
i
\end{array}\right) \lambda^{i} D_{n-i}(Q)
$$

Obviously $D_{n}(Q)=\operatorname{det}(Q)$ while $n D_{1}(Q)$ is the trace of $Q$.

We require the following easily-established (see e.g., Petty [P1] fact: Suppose $y_{i j} \in \mathbb{R}^{n}, 1 \leq i \leq N, 1 \leq j \leq n$, and let the positive semi-definite matrices $Q_{j}$, $1 \leq j \leq n$, be defined by

$$
x \cdot Q_{j} x=\sum_{i=1}^{N}\left|x \cdot y_{i j}\right|^{2}, \quad \text { for all } x \in \mathbb{R}^{n},
$$

then the mixed discriminant of $Q_{1}, \ldots, Q_{n}$ is given by

$$
D\left(Q_{1}, \ldots, Q_{n}\right)=\frac{1}{n !} \sum_{1 \leq i_{1}, \ldots, i_{n} \leq N}\left[y_{i_{1} 1}, \ldots, y_{i_{n} n}\right]^{2} .
$$


It follows immediately from (2.2) that if for non-negative measures $\mu_{1}, \ldots, \mu_{n}$ on $S^{n-1}$, the positive semi-definite matrices $Q_{j}, 1 \leq j \leq n$, are defined by

$$
u \cdot Q_{j} u=\int_{S^{n-1}}|u \cdot v|^{2} d \mu_{j}(v), \quad \text { for all } u \in S^{n-1},
$$

then the mixed discriminant of $Q_{1}, \ldots, Q_{n}$ is given by

$$
D\left(Q_{1}, \ldots, Q_{n}\right)=\frac{1}{n !} \int_{S^{n-1}} \cdots \int_{S^{n-1}}\left[v_{1}, \ldots, v_{n}\right]^{2} d \mu_{1}\left(v_{1}\right) \cdots d \mu_{n}\left(v_{n}\right) .
$$

From this we obtain

Lemma 2.4. Suppose $u_{1}, \ldots, u_{N} \in S^{n-1}$ and $\lambda_{1}, \ldots, \lambda_{N}>0$. If $Q$ is a positive definite matrix so that,

$$
u \cdot Q u=\sum_{i=1}^{N} \lambda_{i}\left|u \cdot u_{i}\right|^{2}, \quad \text { for all } u \in S^{n-1},
$$

then, for $1 \leq k \leq n$,

$$
D_{k}(Q)=\frac{(n-k) !}{n !} \sum_{1 \leq i_{1}, \ldots, i_{k} \leq N} \lambda_{i_{1}} \cdots \lambda_{i_{k}}\left[u_{i_{1}}, \ldots, u_{i_{k}}\right]^{2} .
$$

To prove this take $\mu_{1}=\cdots=\mu_{k}$ in (2.3) to be the measure that is concentrated on $u_{1}, \ldots, u_{N}$ with weights $\lambda_{1}, \ldots, \lambda_{N}$, and let $d \mu_{i}(v)=\omega_{n}^{-1} d v$, for $k+1 \leq i \leq n$. (Note that $Q_{i}=I$, for $k+1 \leq i \leq n$ ), and get

$$
D_{k}(Q)=\frac{c_{n, k}}{n !} \sum_{1 \leq i_{1}, \ldots, i_{k} \leq N}\left[u_{i_{1}}, \ldots, u_{i_{k}}\right]^{2} \lambda_{i_{1}} \cdots \lambda_{i_{k}},
$$

where $c_{n, k}$ is given by

$$
c_{n, k}\left[v_{1}, \ldots, v_{k}\right]^{2}=\omega_{n}^{k-n} \int_{S^{n-1}} \ldots \int_{S^{n-1}}\left[v_{1}, \ldots, v_{k}, v_{k+1}, \ldots, v_{n}\right]^{2} d v_{k+1} \cdots d v_{n} .
$$

Since $c_{n, k}$ above is independent of our choice of $Q$ we can compute $c_{n, k}$ most easily by choosing $\left\{u_{1}, \ldots, u_{N}\right\}$ in $(2.5)$ to be the standard orthonormal basis, $\left\{e_{1}, \ldots, e_{n}\right\}$, in $\mathbb{R}^{n}$, and all the $\lambda_{i}=1$ (and thus $Q=I$ ). This immediately shows that

$$
c_{n, k}=(n-k) !
$$

and completes the proof.

Obviously Lemma 2.1 is the special case of Lemma 2.4 when $Q=I$.

\section{The NEW AFFine FUnCtional AND A NEW AFFine ClASS OF POLYTOPES}

Definition 3.1. If $P$ is a convex polytope in $\mathbb{R}^{n}$ which contains the origin in its interior, and $u_{1}, \ldots, u_{N}$ are the outer normal unit vectors to the faces of $P$, with $h_{1}, \ldots, h_{N}$ the corresponding distances of the faces from the origin and $a_{1}, \ldots, a_{N}$ the corresponding areas of the faces, then define $U(P)$ by

$$
U(P)^{n}=\frac{1}{n^{n}} \sum_{u_{i_{1}} \wedge \cdots \wedge u_{i_{n}} \neq 0} h_{i_{1}} \cdots h_{i_{n}} a_{i_{1}} \cdots a_{i_{n}} .
$$


Obviously the functional $U$ is centro-affine invariant in that,

$$
U(\phi P)=U(P), \quad \text { for all } \phi \in \mathrm{SL}(n) .
$$

Since $V(P)=\frac{1}{n} \sum_{i=1}^{N} a_{i} h_{i}$, it follows immediately that

$$
U(P)<V(P) .
$$

As an aside, we observe that $U(P)$ is significantly less than $V(P)$ only if $P$ is highly symmetric and has few faces. For a random polytope with a large number of faces $U(P)$ is very close to $V(P)$. It is this property of the functional $U$ which will make it so useful.

It will be helpful to introduce a new class of convex polytopes in $\mathbb{R}^{n}$. A convex polytope is said to be in the class $\mathcal{P}_{n}$ if for any two sets of $n$ linearly independent vertices of the polytope, say $v_{1}, \ldots, v_{n}$ and $v_{1}^{\prime}, \ldots, v_{n}^{\prime}$, the simplices whose vertices are $0, v_{1}, \ldots, v_{n}$ and $0, v_{1}^{\prime}, \ldots, v_{n}^{\prime}$ have identical volumes. Obviously, this is a centro-affine invariant class in that for $P \in \mathcal{P}_{n}$ and $\phi \in \operatorname{GL}(n)$, we have $\phi P \in \mathcal{P}_{n}$. It is easily seen that both the regular simplex, whose centroid is at the origin, and the regular cross-polytope are in $\mathcal{P}_{n}$. As an aside, we note that it is easily seen that the number of sides, $N$, of a body in $\mathcal{P}_{2}$ is such that $3 \leq N \leq 6$, with all values between 3 and 6 actually assumed. In fact all the bodies in $\mathcal{P}_{2}$ are easily characterized. However, for larger $n$, no trivial description of the bodies in $\mathcal{P}_{n}$ seems likely.

Let $\mathcal{P}_{n}^{*}$ denote the class of polars of the polytopes in $\mathcal{P}_{n}$. Obviously, this is a centro-affine invariant class as well.

\section{Inequalities for Schneider's PROBlem}

We shall establish

Lemma 4.1. If $P$ is a convex polytope in $\mathbb{R}^{n}$ that contains the origin in its interior, then

$$
\left(\frac{n^{n}}{n !}\right)^{\frac{1}{2}} \omega_{n}[U(P) V(P)]^{n / 2} \geq V\left(\Gamma_{-2} P\right) V(\Pi P)
$$

with equality if and only if $P \in \mathcal{P}_{n}^{*}$.

To prove the lemma, suppose $P$ is a convex polytope in $\mathbb{R}^{n}$ that contains the origin in its interior and $u_{1}, \ldots, u_{N}$ denote the outer unit normals of $P$, with $h_{1}, \ldots, h_{N}$ denoting the corresponding distances from the origin to the faces and $a_{1}, \ldots, a_{N}$ the areas of the corresponding faces. Obviously, the support function of $\Pi P$ is given by

$$
h(\Pi P, u)=\frac{1}{2} \sum_{i=1}^{N}\left|u \cdot u_{i}\right| a_{i}, \quad \text { for all } u \in S^{n-1},
$$

and thus by the McMullen-Matheron-Weil formula (1.4) we have

$$
V(\Pi P)=\frac{1}{n !} \sum_{1 \leq i_{1}, \ldots, i_{n} \leq N} a_{i_{1}} \cdots a_{i_{n}}\left[u_{i_{1}}, \ldots, u_{i_{n}}\right] .
$$


Since volume is an SL(n)-invariant functional, in light of (3.2), (1.2), and (1.3), we see that in order to establish the lemma we may assume, without loss of generality, that $\Gamma_{-2} P$ is a ball; i.e.,

$$
\Gamma_{-2} P=\left(\frac{V\left(\Gamma_{-2} P\right)}{\omega_{n}}\right)^{\frac{1}{n}} B
$$

where $B$ denotes the unit ball centered at the origin and, as before, $\omega_{n}=V(B)$.

From (4.3) and definition (1.1) of $\Gamma_{-2}$, we have

$$
\left(\frac{\omega_{n}}{V\left(\Gamma_{-2} P\right)}\right)^{\frac{2}{n}}=\frac{1}{V(P)} \sum_{i=1}^{N}\left|u \cdot u_{i}\right|^{2} \frac{a_{i}}{h_{i}} .
$$

Now Lemma 2.1, with

gives

$$
\lambda_{i}=\frac{a_{i}}{h_{i}}\left(\frac{V\left(\Gamma_{-2} P\right)}{\omega_{n}}\right)^{\frac{2}{n}} \frac{1}{V(P)}
$$

$$
\left(\frac{\omega_{n}}{V\left(\Gamma_{-2} P\right)}\right)^{2}=\frac{1}{n ! V(P)^{n}} \sum_{1 \leq i_{1}, \ldots, i_{n} \leq N} \frac{a_{i_{1}}}{h_{i_{1}}} \cdots \frac{a_{i_{n}}}{h_{i_{n}}}\left[u_{i_{1}}, \ldots, u_{i_{n}}\right]^{2} .
$$

Now (4.4), together with the Hölder inequality, and (4.2) give

$$
\begin{aligned}
\frac{n ! V(P)^{n}}{n^{n} U(P)^{n}} & \left(\frac{\omega_{n}}{V(\Gamma-2 P)}\right)^{2} \\
& =\frac{1}{n^{n} U(P)^{n}} \sum_{u_{i_{1}} \wedge \cdots \wedge u_{i_{n}} \neq 0}\left(\frac{\left[u_{i_{1}}, \ldots, u_{i_{n}}\right]}{h_{i_{1}} \cdots h_{i_{n}}}\right)^{2} h_{i_{1}} \cdots h_{i_{n}} a_{i_{1}} \cdots a_{i_{n}} \\
& \geq\left(\frac{1}{n^{n} U(P)^{n}} \sum_{u_{i_{1}} \wedge \cdots \wedge u_{i_{n}} \neq 0}\left[u_{i_{1}}, \ldots, u_{i_{n}}\right] a_{i_{1}} \cdots a_{i_{n}}\right)^{2} \\
& =\left(\frac{n !}{n^{n}} \frac{V(\Pi P)}{U(P)^{n}}\right)^{2},
\end{aligned}
$$

with equality if and only if

$$
\frac{\left[u_{i_{1}}, \ldots, u_{i_{n}}\right]}{h_{i_{1}} \cdots h_{i_{n}}}
$$

is independent of the choice of the subscripts whenever $u_{i_{1}} \wedge \cdots \wedge u_{i_{n}} \neq 0$. But

$$
\frac{\left[u_{i_{1}}, \ldots, u_{i_{n}}\right]}{h_{i_{1}} \cdots h_{i_{n}}}=\left[u_{i_{1}} \rho_{i_{1}}^{*}, \ldots, u_{i_{n}} \rho_{i_{n}}^{*}\right]
$$

where $u_{i_{j}} / h_{i_{j}}=u_{i_{j}} \rho_{i_{j}}^{*}$ are the vertices of $P^{*}$ and $\left[u_{i_{1}} \rho_{i_{1}}^{*}, \ldots, u_{i_{n}} \rho_{i_{n}}^{*}\right]$ is equal to $n$ ! times the volume of the simplex whose vertices are $0, u_{i_{1}} \rho_{i_{1}}^{*}, \ldots, u_{i_{n}} \rho_{i_{n}}^{*}$. Thus equality is possible if and only if $P \in \mathcal{P}_{n}^{*}$. This completes the proof.

The following lemma, proved in [LYZ], will be needed.

Lemma 4.5. If $P$ is an origin-symmetric convex polytope in $\mathbb{R}^{n}$, then

$$
V\left(\Gamma_{-2} P\right) \geq 2^{-n} \omega_{n} V(P),
$$

with equality if and only if $P$ is a parallelotope.

This together with Lemma 4.1 immediately gives 
Theorem 4.6. If $P$ is an origin-symmetric convex polytope in $\mathbb{R}^{n}$, then

$$
\frac{V(\Pi P)}{U(P)^{\frac{n}{2}} V(P)^{\frac{n}{2}-1}} \leq 2^{n}\left(\frac{n^{n}}{n !}\right)^{\frac{1}{2}}
$$

with equality if and only if $P$ is a parallelotope.

An immediate consequence of this and (3.3) is

Corollary 4.7. If $K$ is a convex body in $\mathbb{R}^{n}$ that is symmetric about some point, then

$$
\frac{V(\Pi K)}{V(K)^{n-1}} \leq 2^{n}\left(\frac{n^{n}}{n !}\right)^{\frac{1}{2}} .
$$

Reisner's inequality [R1], R2], GMR states that if $K$ is a projection body in $\mathbb{R}^{n}$, then

$$
V(K) V\left(K^{*}\right) \geq \frac{4^{n}}{n !}
$$

The best lower bound for the centro-affine volume product $V(K) V\left(K^{*}\right)$, as $K$ ranges over the class of origin-symmetric convex bodies, is unknown. The best results to date are those of Bourgain and Milman [BoM].

Theorem 4.6 together with Reisner's inequality immediately gives

Theorem 4.8. If $P$ is an origin-symmetric convex polytope in $\mathbb{R}^{n}$, then

$$
V\left(\Pi^{*} P\right) U(P)^{\frac{n}{2}} V(P)^{\frac{n}{2}-1} \geq \frac{2^{n}}{\left(n^{n} n !\right)^{\frac{1}{2}}},
$$

with equality if and only if $P$ is a parallelotope.

From Theorem 4.8 and (3.3) we immediately get

Corollary 4.9. If $K$ is a convex body in $\mathbb{R}^{n}$ that is symmetric about some point, then

$$
V\left(\Pi^{*} K\right) V(K)^{n-1} \geq \frac{2^{n}}{\left(n^{n} n !\right)^{\frac{1}{2}}} .
$$

The problem of determining the best lower bound for the affine product

$$
\left[V\left(\Pi^{*} K\right) V(K)^{n-1}\right]^{1 / n},
$$

as $K$ ranges over the class of origin-symmetric bodies is open and important. The best upper bound for the affine product $V\left(\Pi^{*} K\right) V(K)^{n-1}$, as $K$ ranges over the class of all convex bodies, is given by the Petty projection inequality [P3]. The best lower bound for the affine product $V\left(\Pi^{*} K\right) V(K)^{n-1}$, as $K$ ranges over the class of all convex bodies, is given by the Zhang projection inequality $\mathrm{Z}$. (See also e.g., the books of Schneider [S2], Leichtweiß [Le], and Gardner [G].)

That the inequality of Corollary 4.9 provides an asymptotically optimal lower bound for the affine product $\left[V\left(\Pi^{*} K\right) V(K)^{n-1}\right]^{1 / n}$, as $K$ ranges over the class of origin-symmetric bodies, may be seen by taking $K$ to be the cube.

The following result was established in [LYZ:

Lemma 4.10. If $P$ is a convex polytope in $\mathbb{R}^{n}$ that has its John point at the origin, then

$$
V\left(\Gamma_{-2} P\right) \geq \frac{n ! \omega_{n}}{n^{\frac{n}{2}}(n+1)^{\frac{n+1}{2}}} V(P),
$$

with equality if and only if $P$ is a simplex. 
Together with Lemma 4.1, this gives

Theorem 4.11. If $P$ is a convex polytope in $\mathbb{R}^{n}$ that has its John point at the origin, then

$$
\frac{V(\Pi P)}{U(P)^{\frac{n}{2}} V(P)^{\frac{n}{2}-1}} \leq \frac{n^{n}(n+1)^{\frac{n+1}{2}}}{(n !)^{\frac{3}{2}}},
$$

with equality if and only if $P$ is a simplex.

From this and (3.3) we have

Corollary 4.12. If $K$ is a convex body in $\mathbb{R}^{n}$, then

$$
V(\Pi K) / V(K)^{n-1} \leq n^{n}(n+1)^{\frac{n+1}{2}} /(n !)^{\frac{3}{2}} .
$$

Theorem 4.11 immediately gives Corollary 4.12 for polytopes whose John point is at the origin. But both $V$ and $\Pi$ are translation invariant, which shows that the inequality of Corollary 4.12 holds for arbitrary polytopes. Since both $V$ and $\Pi$ are continuous on the space of convex bodies, with the Hausdorff topology, an obvious approximation argument shows that the inequality of Corollary 4.12 must hold for all convex bodies.

That the inequality of Corollary 4.12 provides an asymptotically optimal bound for the affine ratio $(*)$ can be seen by taking $K$ to be the simplex.

\section{EXtensions of BALl's REVERSE ISOPERIMETRIC INEQUALITY}

Definition 5.1. If $P$ is a convex polytope in $\mathbb{R}^{n}$ which contains the origin in its interior, and $u_{1}, \ldots, u_{N}$ are the outer normal unit vectors to the faces of $P$, with $h_{1}, \ldots, h_{N}$ the corresponding distances of the faces from the origin and $a_{1}, \ldots, a_{N}$ the corresponding areas of the faces, then for $1 \leq j \leq n$, define $U_{j}(P)$ by

$$
U_{j}(P)^{j}=\frac{1}{n^{j}} \sum_{u_{i_{1}} \wedge \cdots \wedge u_{i_{j}} \neq 0} h_{i_{1}} \cdots h_{i_{j}} a_{i_{1}} \cdots a_{i_{j}} .
$$

Obviously, $U_{1}(P)=V(P)$ and $U_{n}(P)=U(P)$. The functional $U_{j}$ is a centroaffine invariant: For each polytope $P$,

$$
U_{j}(\phi P)=U_{j}(P), \quad \text { for all } \phi \in S L(n) .
$$

Ball $\left[\mathrm{B}\right.$ ] proved that an origin-symmetric polytope $P$ in $\mathbb{R}^{n}$ that has been GL $(n)$ transformed into its John position satisfies the following reverse isoperimetric inequality:

$$
\left(\frac{S(P)}{2 n}\right)^{n} \leq V(P)^{n-1}
$$

A convex polytope $P$ was defined in [LYZ to be in dual isotropic position if $\Gamma_{-2} P$ is a ball and $V(P)=1$. Note that for each convex polytope $P$, that contains the origin in its interior, there is a $\mathrm{GL}(n)$ transformation of $P$ that transforms $P$ into a polytope in dual isotropic position.

From the fact that $V_{1}(\Pi P)=2 V_{n-1}(P)=S(P)$, for every polytope $P$, one immediately sees that the inequality of the next theorem, for $j=1$, is precisely Ball's symmetric reverse isoperimetric inequality. 
Theorem 5.2. If $P$ is an origin-symmetric convex polytope in $\mathbb{R}^{n}$ that has been $\mathrm{GL}(n)$-transformed into dual isotropic position, then

$$
\frac{V_{j}(\Pi P)}{U_{j}(P)^{\frac{j}{2}} V(P)^{\frac{j}{2}-\frac{j}{n}}} \leq \frac{2^{j}}{j !}\left(\frac{n ! n^{j}}{(n-j) !}\right)^{\frac{1}{2}}, \quad 1 \leq j<n,
$$

with equality if and only if $P$ is a cube.

Thus Ball's symmetric reverse isoperimetric inequality will hold when the polytope $P$ is in dual isotropic position (as well as in John position).

Ball [B] proved that each polytope $P$ in $\mathbb{R}^{n}$ that has been $\mathrm{GL}(n)$-transformed into its John position satisfies the reverse isoperimetric inequality:

$$
\frac{S(P)^{n}}{V(P)^{n-1}} \leq n^{3 n / 2}(n+1)^{(n+1) / 2} / n !
$$

Our next theorem (for $j=1$ ) shows that this is also the case if the polytope is $\mathrm{GL}(n)$-transformed into dual isotropic position.

Theorem 5.3. If $P$ is a convex polytope in $\mathbb{R}^{n}$ that has been translated so that its John point is at the origin and $\mathrm{GL}(n)$-transformed so that it is in dual isotropic position, then

$$
\frac{V_{j}(\Pi P)}{U_{j}(P)^{\frac{j}{2}} V(P)^{\frac{j}{2}-\frac{j}{n}}} \leq \frac{n^{j}(n !)^{\frac{1}{2}-\frac{j}{n}}(n+1)^{\frac{(n+1) j}{2 n}}}{j ![(n-j) !]^{\frac{1}{2}}}, \quad 1 \leq j<n,
$$

with equality if and only if $P$ is a regular simplex.

Again, note that Theorem 5.3 shows that Ball's reverse isoperimetric inequality will hold when the polytope $P$ is in dual isotropic position (as well as in John position).

To prove Theorems 5.2 and 5.3 we first suppose that $P$ is a convex polytope in $\mathbb{R}^{n}$ that contains the origin in its interior with $u_{1}, \ldots, u_{N}$ the outer unit normals of $P$, with $h_{1}, \ldots, h_{N}$ the corresponding distances from the origin to the faces and $a_{1}, \ldots, a_{N}$ the areas of the corresponding faces. Since the support function of $\Pi P$ is given by

$$
h(\Pi P, u)=\frac{1}{2} \sum_{i=1}^{N}\left|u \cdot u_{i}\right| a_{i},
$$

from the McMullen-Matheron-Weil formula for the intrinsic volume of zonotopes, (1.4), we have

$$
V_{j}(\Pi P)=\frac{1}{j !} \sum_{1 \leq i_{1}, \ldots, i_{j} \leq N}\left[u_{i_{1}}, \ldots, u_{i_{j}}\right] a_{i_{1}} \cdots a_{i_{j}} .
$$

Since $\Gamma_{-2} P$ is defined, for $u \in S^{n-1}$, by

$$
h\left(\Gamma_{-2}^{*} P, u\right)^{2}=\frac{1}{V(P)} \sum_{i=1}^{N}\left|u \cdot u_{i}\right|^{2} \frac{a_{i}}{h_{i}},
$$

and it is assumed that

$$
\Gamma_{-2} P=\left(\frac{V\left(\Gamma_{-2} P\right)}{\omega_{n}}\right)^{\frac{1}{n}} B
$$


and $V(P)=1$, we have

$$
\left(\frac{\omega_{n}}{V\left(\Gamma_{-2} P\right)}\right)^{\frac{2}{n}}=\sum_{i=1}^{N}\left|u \cdot u_{i}\right|^{2} \frac{a_{i}}{h_{i}} .
$$

Now Lemma 2.1, with

$$
\lambda_{i}=\frac{a_{i}}{h_{i}}\left(\frac{V\left(\Gamma_{-2} P\right)}{\omega_{n}}\right)^{\frac{2}{n}}
$$

gives

$$
\left(\frac{\omega_{n}}{V\left(\Gamma_{-2} P\right)}\right)^{\frac{2 j}{n}}=\frac{(n-j) !}{n !} \sum_{1 \leq i_{1}, \ldots, i_{j} \leq N} \frac{a_{i_{1}}}{h_{i_{1}}} \cdots \frac{a_{i_{j}}}{h_{i_{j}}}\left[u_{i_{1}}, \ldots, u_{i_{j}}\right]^{2} .
$$

Now (5.5), together with the Hölder inequality, and (5.4) give (exactly as in the proof of Lemma 4.1):

$$
\frac{1}{n^{j} U_{j}(P)^{j}} \frac{n !}{(n-j) !}\left(\frac{\omega_{n}}{V\left(\Gamma_{-2} P\right)}\right)^{\frac{2 j}{n}} \geq\left(\frac{j !}{n^{j}} \frac{V_{j}(\Pi P)}{U_{j}(P)^{j}}\right)^{2},
$$

with equality if and only if

$$
\frac{\left[u_{i_{1}}, \ldots, u_{i_{j}}\right]}{h_{i_{1}} \cdots h_{i_{j}}}
$$

is independent of the choice of the subscripts whenever $u_{i_{1}} \wedge \cdots \wedge u_{i_{j}} \neq 0$. (Note that if $P$ is a cube centered at the origin, or $P$ is a regular simplex with its centroid at the origin, then this certainly is the case.) Lemma 4.10 (or Lemma 4.5 in the origin-symmetric case) together with the last inequality provide the conclusions of Theorems 5.2 and 5.3.

\section{OPEN PROBLEMS}

Two obvious questions regarding the functionals $V$ and $U$ beg to be asked.

Question 6.1. If $P$ is an origin-symmetric convex polytope in $\mathbb{R}^{n}$, then is it the case that

$$
U(P) \geq n^{-1}(n !)^{1 / n} V(P)
$$

with equality if and only if $P$ is a parallelotope?

Question 6.2. Suppose $P$ is a convex polytope in $\mathbb{R}^{n}$ with its John point at the origin. Is it the case that

$$
U(P) \geq \frac{[(n+1) !]^{\frac{1}{n}}}{n+1} V(P),
$$

with equality if and only if $P$ is a simplex?

We note that the domain of definition of the functional $U$ may be extended (in a natural manner) to include all convex bodies. While we have chosen to present our inequalities only for convex polytopes, all of the inequalities presented in this note hold for arbitrary convex bodies. 


\section{REFERENCES}

[B] K. Ball, Volume ratios and a reverse isoperimetric inequality, J. London Math. Soc. 44 (1991), 351-359. MR 92j:52013

[BoLi] J. Bourgain and J. Lindenstrauss, Projection bodies, Geometric Aspects of Functional Analysis (J. Lindenstrauss and V.D. Milman, Eds.) Springer Lecture Notes in Math. 1317 (1988), 250-270. MR 89g:46024

[BoM] J. Bourgain and V. Milman, New volume ratio properties for convex symmetric bodies in $R^{n}$, Invent. Math. 88 (1987), 319-340. MR 88f:52013

[Br] N.S. Brannen, Volumes of projection bodies, Mathematika 43 (1996), 255-264. MR 98e:52005

[G] R. J. Gardner, Geometric Tomography, Cambridge Univ. Press, Cambridge, 1995. MR 96j:52006

[GiPa] A. Giannopoulos and M. Papadimitrakis, Isotropic surface area measures, Mathematika 46 (1999), 1-14. CMP 2000:10

[GoW] P. R. Goodey and W. Weil, Zonoids and generalizations, Handbook of Convex Geometry (P.M. Gruber and J.M. Wills, Eds.), North-Holland, Amsterdam, 1993, pp. 1297-1326. MR 95g:52015

[GMR] Y. Gordon, M. Meyer, and S. Reisner, Zonoids with minimal volume-product. A new proof, Proc. Amer. Math. Soc. 104 (1988), 273-276. MR 89i:52015

[Le] K. Leichtweiß, Affine Geometry of Convex Bodies, J.A. Barth, Heidelberg, 1998. MR 2000j:52005

[LiM] J. Lindenstrauss and V. D. Milman, Local theory of normed spaces and convexity, Handbook of Convex Geometry (P.M. Gruber and J.M. Wills, Eds.), North-Holland, Amsterdam, 1993, pp. 1149-1220. MR 95b:46012

[L] E. Lutwak, Centroid bodies and dual mixed volumes, Proc. London Math. Soc. 60 (1990), 365-391. MR 90k:52024

[LZ] E. Lutwak and G. Zhang, Blaschke-Santaló inequalities, J. Differential Geom. 47 (1997), 1-16. IMR 2000c:52011

[LYZ] E. Lutwak, D. Yang and G. Zhang, A new ellipsoid associated with convex bodies, Duke Math. J. 104 (2000), 375-390. CMP 2001:01

[Ma] G. Matheron, Random sets and integral geometry, Wiley, New York, 1975. MR 52:6828

[Mc] P. McMullen, Non-linear angle-sum relations for polyhedral cones and polytopes, Math. Proc. Camb. Phil. Soc. 78 (1975), 247-261. MR 52:15238

[MPa1] V. D. Milman and A. Pajor, Cas limites des inégalités du type Khinchine et applications géométriques, C.R. Acad. Sci. Paris 308 (1989), 91-96. MR 90d:52018

[MPa2] V. D. Milman and A. Pajor, Isotropic position and inertia ellipsoids and zonoids of the unit ball of a normed n-dimensional space, Geometric Aspects of Functional Analysis, Springer Lecture Notes in Math. 1376 (1989), 64-104. MR 90g:52003

[P1] C. M. Petty, Centroid surfaces, Pacific J. Math. 11 (1961), 1535-1547. MR 24:A3558

[P2] C. M. Petty, Projection bodies, Proc. Coll. Convexity, Copenhagen, 1965, Københavns Univ. Mat. Inst., 1967, pp. 234-241. MR 35:7203

[P3] C. M. Petty, Isoperimetric problems, Proc. Conf. Convexity and Combinatorial Geometry (Univ. Oklahoma, 1971), University of Oklahoma, 1972, pp. 26-41. MR 50:14499

[R1] S. Reisner, Random polytopes and the volume-product of symmetric convex bodies, Math. Scand. 57 (1985), 386-392. MR 87g:52011

[R2] S. Reisner, Zonoids with minimal volume-product, Math. Z. 192 (1986), 339-346. MR 89i:52015

[S1] R. Schneider, Random hyperplanes meeting a convex body, Z. Wahrscheinlichkeitsth. verw. Geb. 61 (1982), 379-387. MR 84c:60023

[S2] R. Schneider, Convex Bodies: the Brunn-Minkowski Theory, Cambridge Univ. Press, Cambridge, 1993. MR 94d:52007

[SW] R. Schneider and W. Weil, Zonoids and related topics, Convexity and its Applications (P.M. Gruber and J.M. Wills, Eds.), Birkhäuser, Basel, 1983, pp. 296-317. MR 85e:52010

[Sh] G. C. Shephard, Combinatorial properties of associated zonotopes, Canad. J. Math. 24 (1974), 302-321. MR 50:14496

[T] A.C. Thompson, Minkowski Geometry, Cambridge Univ. Press, Cambridge, 1996. MR 97f:52007 
[W] W. Weil, Kontinuierliche Linearkombination von Strecken, Math. Z. 148 (1976), 71-84. MR 53:3887

[Z] G. Zhang, Restricted chord projection and affine inequalities, Geom. Dedicata 39 (1991), 213-222. MR 92f:52017

Department of Mathematics, Polytechnic University, Brooklyn, New York 11201

E-mail address: elutwak@poly.edu

Department of Mathematics, Polytechnic University, Brooklyn, New York 11201

E-mail address: yang@magnus.poly.edu

Department of Mathematics, Polytechnic University, Brooklyn, New York 11201

E-mail address: gzhang@poly.edu 